\title{
Avaliação alométrica e da biomassa de Melanoides tuberculata (MULLER, 1774) como ferramenta para a avaliação da qualidade ambiental de reservatórios
}

\author{
Marconeide de Araújo $^{1}$ (D), Evaldo de Lira Azevêdo ${ }^{2 *}$ (D), Daniele Jovem-Azevêdo ${ }^{3}$ (D), José Etham \\ de Lucena Barbosa ${ }^{4}$ (D), Joseline Molozzi ${ }^{5}$ (D)

\footnotetext{
${ }^{1}$ Universidade Estadual da Paraíba, R. Baraúnas, no 351, Universitário, Campina Grande - PB, Brasil, 58429500.

${ }^{2}$ Unidade de Formação Geral no Instituto Federal de Educação, Ciência e Tecnologia da Paraíba, Campus Cajazeiras, R. José Antônio da Silva, nº 300, Jardim Oásis, Cajazeiras - PB, Brasil, 58900000.

${ }^{3}$ Programa de Pós-Graduação em Ciências Naturais e Biotecnologia da Universidade Federal de Campina Grande, Campus Cuité, Acesso Profa. Maria Anita Furtado Coelho, S/N, Olho D’Água da Bica, Cuité - PB, Brasil, 58175000.

${ }^{4}$ Programa de Pós-Graduação em Ecologia e Conservação e Departamento de Biologia na Universidade Estadual da Paraíba, R. Baraúnas, n 351, Universitário, Campina Grande - PB, Brasil, 58429500.

${ }^{5}$ Programa de Pós-Graduação em Ecologia e Conservação e Departamento de Biologia na Universidade Estadual da Paraíba, R. Baraúnas, n 351, Universitário, Campina Grande - PB, Brasil, CEP 58429500.
} \\ *Autor para correspondência: evaldo.azevedo@ifpb.edu.br
}

Recebido em 09 de abril de 2020.

Aceito em 12 de dezembro de 2020.

Publicado em 31 de dezembro de 2020.

Resumo - Melanoides tuberculata é uma espécie exótica de molusco já disseminada na maioria dos ambientes aquáticos do Brasil. O objetivo desse trabalho foi analisar se há relação das medidas alométricas e da biomassa de $M$. tuberculata com a gradiente de eutrofização em reservatórios. O estudo foi realizado em três reservatórios localizados na bacia do rio Paraíba, Nordeste do Brasil. O índice de estado trófico (IET) de Carlson, modificado por Toledo, foi aplicado para classificação trófica. Os resultados mostram a formação de três grupos entre os locais de amostragem com base no IET. Nos sites com maiores valores de IET foram encontrados os maiores tamanhos do molusco. Enquanto nos sites com níveis médios de eutrofização, os indivíduos tiveram comprimento intermediário. No grupo com a menor média de eutrofização, foram encontramos os menores comprimentos de $M$. tuberculata. A biomassa variou entre os grupos. As medidas alométricas estiveram relacionadas ao gradiente de eutrofização, especialmente quando se considerou, separadamente, as concentrações de fósforo e clorofila. Os resultados obtidos mostram que relações entre medidas alométricas de $M$. tuberculata e variações nas concentrações de fósforo e clorofila apresentam grande potencial para a avaliação da qualidade ambiental de reservatórios no semiárido do Brasil.

Palavras-chave: Degradação Ambiental. Gradiente ambiental. Índice de Estado Trófico. Moluscos Exóticos.

\section{Allometric and Melanoides tuberculata (MULLER, 1774) biomass assessment as a tool for the environmental quality assessment of reservoirs}

Abstract - Melanoides tuberculata is an exotic species already widespread in most aquatic environments in Brazil. The aim of this study was to analyze if there is a relationship between the allometric measures and the M. tuberculata biomass with eutrophication gradient in reservoirs. The 
study was carried out in three reservoirs located in the Rio Paraíba basin, Northeast of Brazil. The Trophic State Index (TSI) of Carlson, modified by Toledo, have been applied for trophic classification. The results show the formation of three groups among the sampling sites based on the TSI. On sites with higher TSI values have been found the highest sizes of M. tuberculata. Whereas in sites with average levels of eutrophication, the individuals had an intermediate shell length. In the group with the lowest average eutrophication, we found the shortest lengths of M. tuberculata. The biomass also varied between the groups. Allometric measurements were related to the eutrophication gradient, especially when phosphorus and chlorophyll concentrations were considered separately. The achieved results show that relations between allometric measures of M. tuberculata and variations in phosphorus and chlorophyll concentrations present great potential for the assessment of the environmental quality of reservoirs in the semiarid region of Brazil.

Keywords: Environmental degradation. Environmental gradient. Trophic State Index. Exotic Mollusks.

\section{Evaluación alométrica y de biomasa de Melanoides tuberculata (MULLER, 1774) como herramienta para la evaluación de la calidad ambiental de reservorios}

Resumen - Melanoides tuberculata es una especie exótica de moluscos ya diseminada en la mayoría de los ambientes acuáticos de Brasil. El objetivo de este trabajo consiste en analizar si hay relación entre las medidas alométricas y de la biomasa de $M$. tuberculata y el gradiente de eutrofización en reservorios. El estudio fue realizado en tres reservorios ubicados en la cuenca del río Paraíba, Nordeste de Brasil. El Índice del Estado Trófico (IET) de Carlson, modificado por Toledo, fue aplicado para clasificación trófica. Los resultados presentan la formación de tres grupos entre los sitios de muestreos con base en el IET. En los sitios con mayores valores de IET se encontraron los tamaños más grandes del molusco. Mientras que en los sitios con niveles medios de eutrofización los individuos tuvieron longitud intermedia. En el grupo con promedio de eutrofización más pequeña, se encontraron las longitudes más pequeñas de $M$. tuberculata. La biomasa ha variado entre los grupos. Las medidas alométricas se relacionaron con el gradiente de eutrofización, sobre todo cuando se consideró en separado las concentraciones de fósforo y clorofila. Los resultados obtenidos muestran que las relaciones entre las medidas alométricas de M. tuberculata y las variaciones en las concentraciones de fósforo y clorofila tienen un gran potencial para la evaluación de la calidad ambiental de los reservorios en la región semiárida de Brasil.

Palabras clave: Degradación Ambiental. Gradiente ambiental. Índice de Estado Trófico. Moluscos Exóticos. 
Avaliação alométrica e da biomassa de Melanoides tuberculata (MULLER, 1774) como ferramenta para a avaliação da qualidade ambiental de reservatórios

\section{Introdução}

Os reservatórios são ecossistemas lênticos que tem a finalidade de armazenar água, garantindo além do abastecimento humano, a dessedentação animal, o uso industrial, a irrigação, o lazer e o turismo (Macedo e Sipaúba-Tavares 2010). Esses ecossistemas possuem um papel ainda mais importante em regiões onde a escassez hídrica é dominante, como as áreas áridas e semiáridas, onde atuam como a principal fonte de recursos hídricos para o desenvolvimento social e econômico (Liebe et al. 2005; Rezende et al. 2018). No entanto, os reservatórios sofrem com o processo de eutrofização, fenômeno caracterizado pelo aumento significativo de nutrientes na água, ocasionado, principalmente por ação antrópica, como pela liberação de efluentes urbanos, fertilizantes agrícolas e esgoto doméstico (Dodds e Smith et al. 2006; Barbosa et al. 2012).

No semiárido brasileiro os períodos prolongados de estiagem contribuem diretamente para a eutrofização, causando a redução do nível pluviométrico e elevadas taxas de evapotranspiração, que em conjunto promovem a redução do volume hídrico e o aumento na concentração de nutrientes nos reservatórios (Araújo 2011; Braga et al. 2015; Menezes et al. 2019). O estado eutrófico favorece a proliferação de cianobactérias, entre as quais estão organismos que são capazes de produzir e liberar toxinas prejudiciais à saúde humana e de outros animais (Oliver e Ribeiro 2014; da Costa et al. 2016). Desse modo, causando prejuízos à qualidade da água, o que afeta diversos serviços ecossistêmicos fornecidos pelos reservatórios, como o abastecimento domiciliar de água potável, a recreação, o valor estético, o potencial turísitco, a pesca e a conservação da biodiversidade (Salameh e Harahsheh, 2011; Reynaud e Lanzanova, 2017). Assim, para promover o refinamento das políticas de gestão ambiental é necessário adotar ferramentas que avaliem a eutrofização desses ecossistemas aquáticos (Junior et al. 2018).

A eutrofização também promove o desaparecimento de espécies nativas especialistas, favorecendo o estabelecimento de espécies oportunistas, como as exóticas, as quais são frequentemente tolerantes às condições ambientais de degradação (Salameh e Harahsheh, 2011). Melanoides tuberculata (Muller 1774), é um exemplo de espécie exótica amplamente distribuída nos reservatórios do semiárido brasileiro (Eskinazi-Sant'Anna et al. 2006), que vem apresentando seus maiores índices de reprodução em locais degradados (Molozzi et al. 2013; Azevêdo et al. 2015; Paiva et al. 2018). Trata-se de um molusco pertencente à família Thiaridae, de origem Asiática conhecido por apresentar elevada capacidade adaptativa e dispersiva, sendo capaz de se estabelecer nos mais diversos substratos (Santos e Eskinazi-Sant'Anna, 2010).

Esse molusco apresenta alta plasticidade ecológica, podendo ser encontrado em ambientes oligotróficos, mesotróficos e eutróficos (Santos e Eskinazi-Sant’Anna 2010). Devido a essa característica e pelo fato de fornecer respostas associadas ao gradiente de impacto ambiental, como pelo aumento ou redução da densidade populacional (Queiroz, 2008), consideramos que esta espécie pode ser utilizada como bioindicadora da qualidade ambiental em reservatórios brasileiros. Tendo em vista que bioindicadores ambientais são caracterizados como grupos de organismos capazes de refletir a integridade dos ecossistemas através de suas respostasas e modificações nas condições ambientais (Barbour et al. 1999), sendo amplamente usados em estudos de monitoramento da saúde dos ecossistemas (Goulart e Callisto, 2003).

Assim, propomos a bioavaliação dos reservatórios através da análise das medidas alométricas, comprimento e abertura da concha de M. tuberculata, pois estas medidas poderão indicar o 
grau de trofia dos corpos hídricos. Isso porque, o nível de desenvolvimento dos moluscos nos diferentes níveis de trofia, assim como a biomassa da comunidade, podem refletir a capacidade de acumulação de energia advindos da interação com o meio. Nesse contexto, o presente estudo teve o objetivo de analisar a relação das medidas alométricas (comprimento e abertura da concha) e da biomassa de $M$. tuberculata com o gradiente de eutrofização em reservatórios no semiárido.

\section{Material e métodos}

\section{Área de estudo e desenho amostral}

A região semiárida do Brasil é caracterizada principalmente por apresentar temperaturas elevadas (acima de $20^{\circ} \mathrm{C}$ ) e precipitações escassas (entre 280 a $800 \mathrm{~mm} /$ ano) (Araújo2011). Isso estabelece a variação dos elementos que compõem a paisagem da região. Por conta dessas características os rios são intermitentes e, consequentemente, os reservatórios apresentam elevado tempo de residência da água (Wiegandet al. 2016). O que torna esses ecossistemas mais susceptíveis ao aumento das concentrações de nutrientes e eutrofização (Braga et al. 2015).

Neste estudo foram avaliados três reservatórios: Acauã (Argemiro de Figueiredo) (253.000.000 m3 - capacidade máxima de acumulação), Poções (29.861.562 m3) e Boqueirão (Epitácio Pessoa) (411.686.287 m3), os quais pertencem à bacia hidrográfica do rio Paraíba (Figura 1). Esses reservatórios são reconhecidos por possuírem múltiplos usos, tais como abastecimento da população, irrigação e lazer (Azevêdoet al. 2017a). A eutrofização à qual estão sujeitos acarreta grandes prejuízos à disponibilidade dos serviços ecossistêmicos prestados por esses reservatórios (Barbosa et al. 2012), gerando problemas como contaminação por cianotoxinas, o encarecimento dos serviços de tratamento de água, a redução da produção de peixes, assim como a presença de sabores e odores desagradáveis na água (Salameh e Harahsheh, 2011).

As amostragens foram realizadas em cinco períodos entre os anos de 2015 e 2016: julho e outubro de 2015 e, fevereiro, junho e outubro de 2016. Em cada reservatório foram estabelecidas três zonas: Região Próxima ao Principal Rio Tributário (RPT), Região Intermediária (RI) e Região Próxima ao Barramento (RPB), onde foram selecionados três sites de amostragem em cada zona (Figura 1). 
Figura 1. Mapa com a localização dos três reservatórios, bacia hidrográfica do rio Paraíba Brasil. ${ }^{*} \mathrm{RPT}$ $=$ Região Próxima ao Principal Rio Tributário; RI = Região Intermediária; RPB = Região Próxima ao

Barramento.

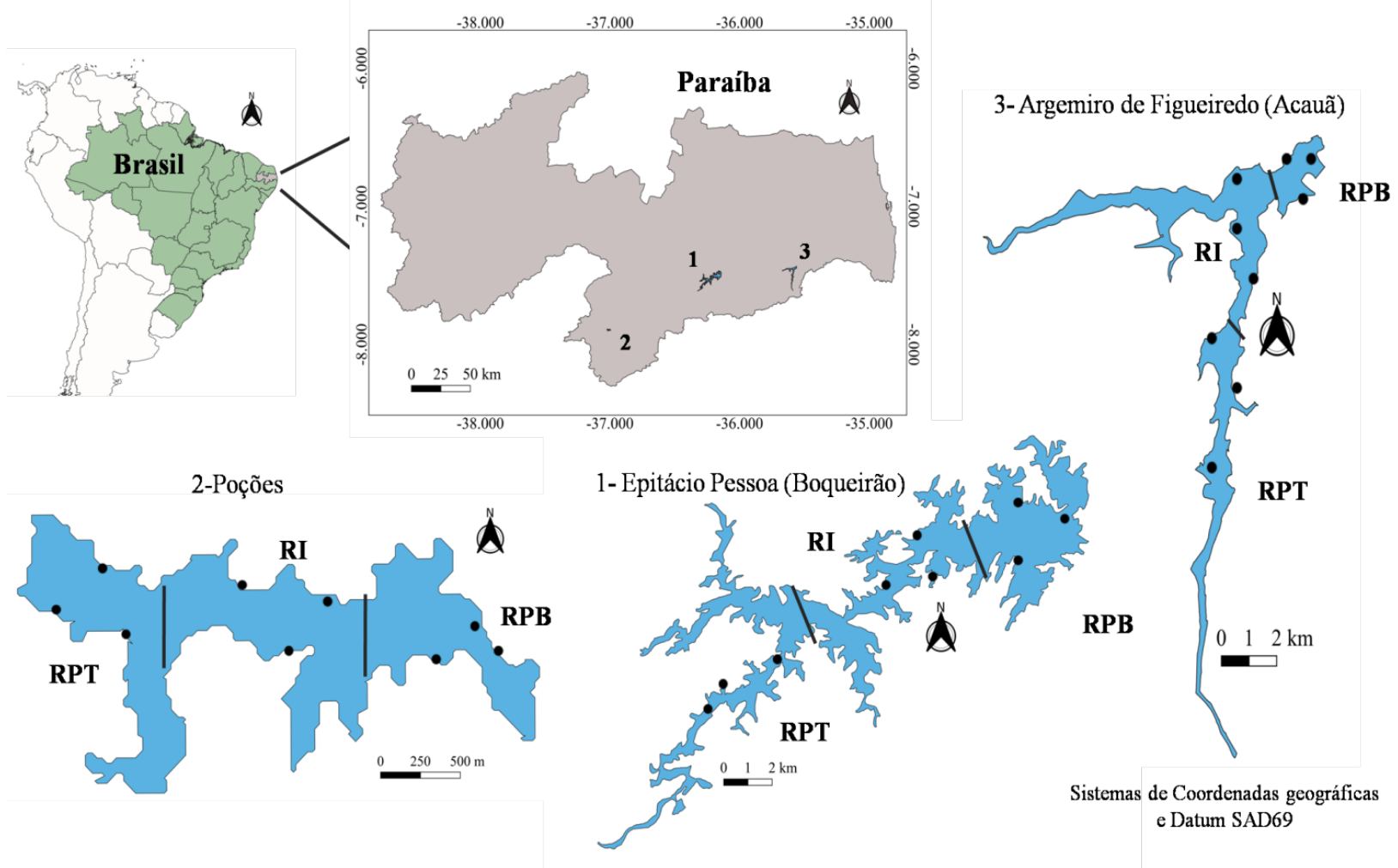

\section{Variáveis abióticas e Índice de Estado Trófico}

Para a avaliação dos parâmetros abióticos foi coletado 1 litro de água na sub-superfície do corpo aquático, em cada um dos nove locais de amostragem durante os cinco períodos. In situ, a transparência da água foi avaliada de acordo com o desaparecimento do disco de Secchi na coluna d'água em cada site de amostragem. Em laboratório, foram realizadas análises para estimativa da concentração de clorofila-a $(\mu \mathrm{g} / \mathrm{L})$, fósforo total $(\mu \mathrm{g} / \mathrm{L})$, fosfato solúvel reativo $(\mu \mathrm{g} / \mathrm{L})$, de acordo com os procedimentos descritos em APHA (2005). Posteriormente, os dados abióticos obtidos foram utilizados para o cálculo do Índice de Estado Trófico (IET). Aqui, selecionamos o índice de estado trófico de Carlson (1977) (IET), elaborado para regiões temperadas e modificado por Toledo Jr. et al. (1983). Segundo critérios do índice, valores de IET < 44 indicam ambiente oligotrófico; 44 $<$ IET < 54, mesotrófico e IET > 54, eutrófico.

\section{Coleta de M. tuberculata, avaliação alométrica e de biomassa}

Em cada um dos nove locais de amostragem, durante os cinco períodos, foi coletada uma amostra de sedimento utilizando uma draga Erkman-Birge $\left(225 \mathrm{~cm}^{2}\right)$. O sedimento foi acondicionado em sacos plásticos e fixado com formaldeído a 4\%. Em laboratório, o material foi lavado e triado para retirada dos moluscos. Os indivíduos encontrados foram contados e identificados utilizando chave de identificação especializada (Mugnai et al. 2010). 
A avaliação alométrica de $M$. tuberculata foi realizada através da medição do comprimento e da abertura da concha. Para isso, a concha foi mensurada a partir do ápice à extremidade de sua abertura, utilizando um paquímetro. Indivíduos com menor tamanho foram mensurados utilizando uma régua microscópica. Para a abertura da concha foi mensurado o seu diâmetro, considerando a face que forneceu a maior medida.

Para avaliação da biomassa os organismos foram secos em estufa a $60^{\circ} \mathrm{C}$ durante $72 \mathrm{~h}$ e depois pesados em balança de precisão (10-5) para estimar o peso seco. Posteriormente, os moluscos foram incinerados em forno do tipo mufla a $450^{\circ} \mathrm{C}$ durante $4 \mathrm{~h}$ para estimar o peso seco livre de cinzas. Finalmente, o valor da biomassa foi obtido a partir da diferença entre o peso da amostra seca e o peso incinerado.

\section{Análises de dados}

Foi realizada uma análise de agrupamento (Cluster analysis) para avaliar a segregação entre os locais de amostragem, considerando o nível de eutrofização através do Índice de Estado Trófico (IET). Essa análise foi aplicada a partir da matriz de dissimilaridade utilizando a distância Euclidiana. Para avaliar diferenças entres grupos formados a partir do IET, foi realizada análise de significância com 9999 permutações (Permutational Analysis of Variance - PERMANOVA; Anderson 2001; Anderson et al. 2008). Essa análise foi realizada utilizando o pacote estatístico PRIMER- 6 + PERMANOVA (Anderson et al. 2008).

Outra série de PERMANOVA's foi aplicada para analisar as diferenças existentes entre os grupos formados a partir do IET em relação ao tamanho e abertura da concha (matriz de Distância Euclidiana; 9999 permutações) e, biomassa (matriz de dissimilaridade de Bray-Curtis; 9999 permutações). Para visualizar o comportamento dos dados de biomassa, tamanho e abertura da concha do molusco, foram construídos gráficos box-plots. Por fim, para correlacionar os dados de IET, concentrações de fósforo e clorofila, com as medidas alométricas e de biomassa foi realizada análise de correlação de Pearson $(<0,5)$. Para essas análises foi utilizado o programa BioEstat 5.0 (Ayres et al. 2007).

\section{Resultados}

De acordo com o IET, os três reservatórios estudados encontram-se classificados como eutróficos. A análise de agrupamento (CLUSTER) resultou na formação de três grupos entre os locais de amostragem, aqui denominados: grupo 1 (maiores valores de IET), grupo 2 (valores intermediários de IET) e grupo 3 (menores valores de IET) (Figura 2). Houve diferença significativa entre os grupos formados (PERMANOVA: Pseudo $\mathrm{F}_{2,75}=29.05, \mathrm{p}=0,0001$ ). Os maiores valores para o IET foram encontrados para o grupo $1(83.62, \pm 4.59)$ e menores valores para o grupo 3 $(60.18, \pm 4.69)$ durante o período de estudo. Todos os demais dados (tamanho do molusco, abertura da concha, biomassa e abundância) foram analisados considerando o mesmo agrupamento formado a partir dos dados de IET. 
Figura 2. Distribuição dos grupos de acordo com o Índice de Estado Trófico (IET). Sendo: grupo $1=$ maiores valores de IET; grupo 2 = valores intermediários de IET e; grupo 3 = menores valores de IET.

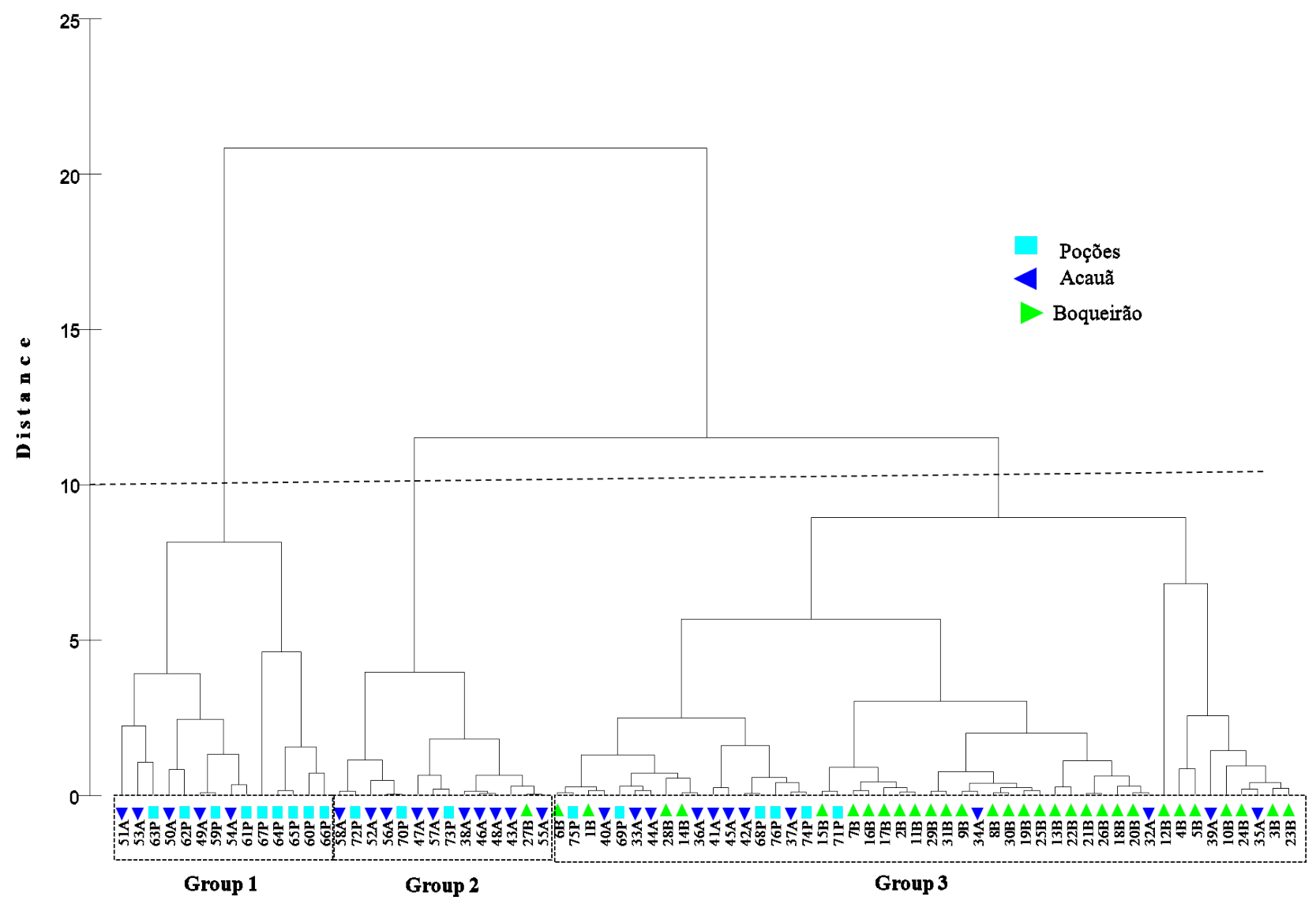

Um total de 13.286 indivíduos da espécie $M$. tuberculata foram medidos, o grupo três apresentou a maior abundância com 7.640 indivíduos; seguido pelo grupo dois com 4.423 indivíduos, e o grupo um, que apresentou a menor abundância entre os três, com 1.223 indivíduos.

Os tamanhos dos moluscos variaram de $2 \mathrm{a} 17 \mathrm{~mm}$. Houve diferença significativa dos tamanhos dos organismos entre os grupos (PERMANOVA: Pseudo $\mathrm{F}_{2,75}=4.64, \mathrm{p}=0,01$ ). Considerando a abertura da concha, os diâmetros variaram entre 1 e $7 \mathrm{~mm}$, assim como para o tamanho dos organismos, diferença significativa também foi encontrada para a abertura da concha dos organismos (PERMANOVA: Pseudo $\mathrm{F}_{2,75}=4.64, \mathrm{p}=0,01$ )

No grupo 1 ocorreram os maiores valores de comprimento (16,83 $\mathrm{mm}$ de comprimento máximo; $11,85 \pm 2,40$ de comprimento médio) e de abertura da concha $(4,28 \mathrm{~mm} ; 3,56, \pm$ 0,070). No grupo 2, os tamanhos intermediários foram encontrados tanto para o comprimento $(14,73 \mathrm{~mm} ; 10,48 \pm 1,98)$, quanto para a abertura da concha $(4,18 \mathrm{~mm} ; 3,18, \pm 0,49)$ do gastrópode. Enquanto no grupo 3, ocorreram os menores valores para o comprimento (14,63 $\mathrm{mm} ; 9,68, \pm 2,45)$ e abertura da concha $(4,5 \mathrm{~mm} ; 3,05, \pm 0,68)$ de M. tuberculata (Figura 3). Considerando a biomassa de $M$. tuberculata foi possível observar diferença significativa entre os grupos (PERMANOVA: Pseudo $\mathrm{F}_{2,75}=3.80, \mathrm{p}=0,02$ ). Os valores de biomassa variaram entre 0,03 e 48,18g. O grupo 1 apresentou biomassa média de 5,41g ( $\pm 10,82)$, o grupo 2 de $15,94 \mathrm{~g}( \pm 15,57)$, e o grupo 3 de $7.37 \mathrm{~g}( \pm 9,91)$ (Figura 3$)$. 
Figura 3. Valores de tamanho, abertura da concha, biomassa e, abundância de Melanoides tuberculata nos três grupos de locais de amostragem.
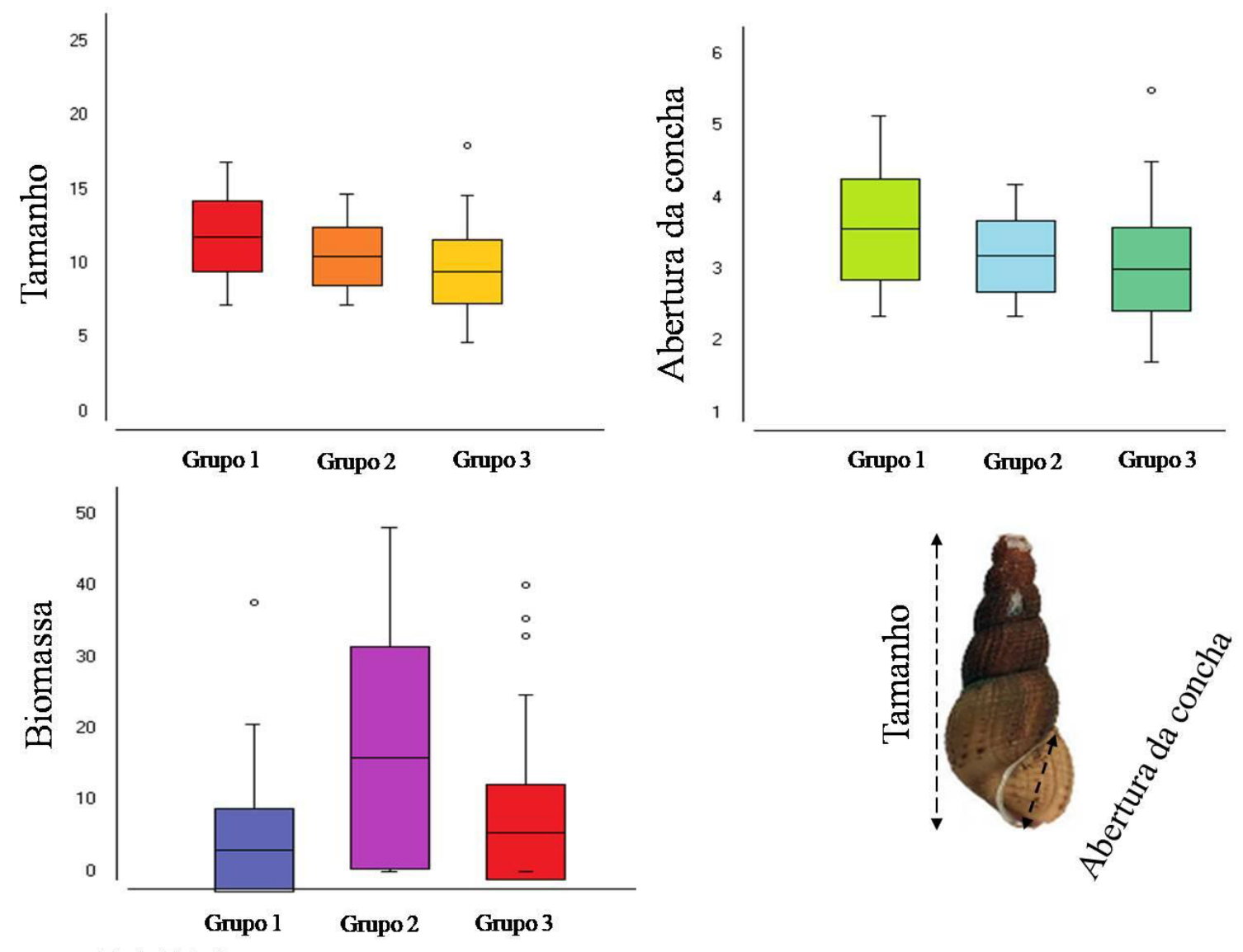

Fonte: Próprios autores.

Correlações positivas significativas ocorreram quando comparado o comprimento de $M$. tuberculata com as concentrações de fósforo total $(\mathrm{r}=0,28 ; \mathrm{p}=0,01)$ e clorofila- $a(\mathrm{r}=0,24 ; \mathrm{p}=$ 0,03) (Fig. 4). Significância também foi observada nas correlações positivas que foram encontradas entre a abertura da concha com as concentrações de fósforo total $(r=0,25 ; p=0,02)$ (Fig. 4). No entanto, correlações fracas e não significativas, foram estabelecidas entre do comprimento com IET $(\mathrm{r}=0,15)$ e, abertura da concha com clorofila- $a(\mathrm{r}=0,17)(\mathrm{p}>0,05)$. 
Avaliação alométrica e da biomassa de Melanoides tuberculata (MULLER, 1774) como ferramenta para a avaliação da qualidade ambiental de reservatórios

Figura 4. Modelo simplificado para compressão da variação das medidas alométricas de M. tuberculata em relação a eutrofização.

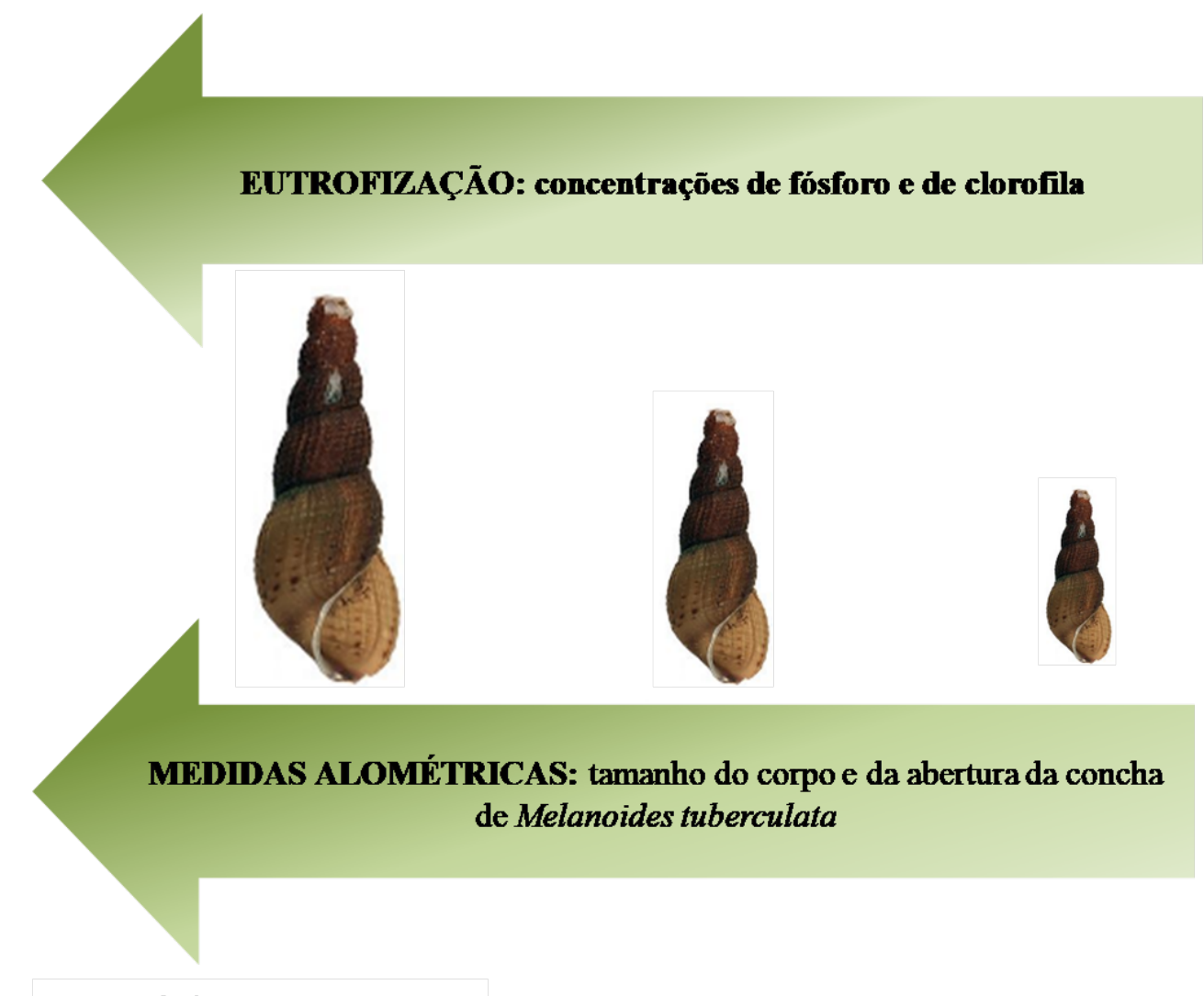

Fonte: Próprios autores.

\section{Discussão}

Melanoides tuberculata é uma espécie exótica já disseminada nos ecossistemas aquáticos do semiárido brasileiro, dominando facilmente ambientes degradados (Eskinazi-Sant'Anna et al. 2006; Azevêdo et al. 2015). Alguns estudos têm mostrado que esse molusco tem preferência por ambientes eutrofizados (Azevêdo et al. 2017a; De Sousa Souto et al. 2011), o que explica sua presença nos três reservatórios estudados, os quais apresentaram elevado nível de eutrofização. Mesmo com alto nível de eutrofização em todos os locais amostrados, foi possível registrar variações das medidas alométricas de $M$. tuberculata entre os ambientes. Os maiores organismos foram registrados no grupo 1, o qual agrupa os sites com os maiores valores de IET.

A biomassa de M. tuberculata também varia amplamente em relação ao gradiente de eutrofização, apresentando valores elevados em ambientes degradados devido a sua plasticidade ecológica (Rawet al. 2016). Em nosso estudo, a biomassa apresentou diferenças marcantes em relação aos grupos. Esperava-se que os maiores valores de biomassa fossem registrados no grupo 1, o mesmo grupo em que estão englobados os organismos com maiores tamanhos, e maiores valores de IET. No entanto, os resultados mostraram uma maior biomassa no grupo 2 e valores intermediários no grupo 3, e menores valores no grupo 1 .

Embora as medidas alométricas tenham variado de acordo com o IET, as correlações estabelecidas foram fracas e sem significância. No entanto, quando analisamos as concentrações de fósforo e 
clorofila individualmente, observa-se o estabelecimento de correlações moderadas significativas com as medidas de comprimento e abertura da concha, indicando resultados mais consistentes para a indicação da qualidade ambiental dos reservatórios. A avaliação das concentrações de fósforo, nitrogênio e clorofila- $a$ são variáveis que refletem a produtividade do ecossistema, fator que influencia diretamente no estabelecimento de espécies tolerantes à degradação ambiental (Trindade e Mendonça, 2014). Nesse sentido, com a disponibilidade de nutrientes e de recursos alimentares, como matéria orgânica e perifiton (Vasconcelos et al. 2013), o molusco pode investir no aumento do seu tamanho. Outros fatores que podem ter promovido o aumento do tamanho, pode ter sido a baixa competitividade, favorecida pela alta disponibilidade de alimento, também observada a partir das concentrações de clorofila- $a$, o que possibilita a melhor sobrevivência da espécie. Estudos prévios em reservatórios do semiárido indicam que esse molusco exótico domina frequentemente a fauna bentônica desses ecossistemas (Eskinazi-Sant'Anna et al. 2006; Azevêdo et al. 2015; Azevêdo et al. 2017b; Oliveira et al. 2019).

De acordo com os dados avaliados, é possível considerar que a qualidade ambiental de reservatórios pode ser mensurada considerando a relação das medidas alométricas (comprimento e abertura da concha) de $M$. tuberculata e variáveis abióticas, como concentrações de fósforo, clorofila- $a$ e gradiente de eutrofização. Nesse sentido, essa abordagem pode ser aplicada como uma ferramenta diagnóstica da eutrófização de reservatórios. Possibilitando o estabelecimento de medidas que auxiliam o controle desse processo, o qual traz grandes danos a esses ecossistemas aquáticos, cruciais para a manutenção da sociedade e economia. A ausência de controle da eutrofização, causa prejuízos no fornecimento de serviços ecossistêmicos essenciais, tais como abastecimento para consumo humano e animal, produção de peixes, e lazer (Salameh e Harahsheh, 2011; Barbosa et al. 2012). Ainda, a eutrofização possui um papel relevante no âmbito da saúde pública, tendo em vista que favorece a produção de cianotoxinas, as quais têm sido encontradas em reservatórios de abastecimento de água no nordeste brasileiro (Fonseca et al. 2015; Lorenzi et al. 2018). Essas toxinas são reconhecidas pelos prejuízos que podem acarretar a saúde humana (Drobacet al. 2013), devido a muitas apresentarem neurotoxicidade, hepatotoxicidade e possuírem a potencialidade de promover o desenvolvimento de tumores (Zhou et al. 2002; van Apeldoorn et al. 2007; Ren et al. 2017). Esse fato se torna ainda mais preocupante diante da realidade em ambientes de regiões áridas e semiáridas, onde muitas vezes os moradores das redondezas dos reservatórios consomem essa água sem nenhum tratamento prévio, havendo ainda a venda e distribuição desse recurso sem tratamento adequado (Azevêdo et al. 2017a).

O desenvolvimento de ferramentas para a avaliação da qualidade da água é crucial para definir o uso desse recurso (Pompêo 2017). De modo geral, embora todos os locais de amostragem estivessem passando por elevado estresse ambiental no período de estudo, nossos resultados indicam que há variação nas medidas alométricas da espécie exótica em função da variação do gradiente de eutrofização (Figura 4). Isso pode estar associado ao fato que as variáveis determinantes para o gradiente de eutrofização (variáveis abióticas) estão relacionadas a modelagem do nicho das espécies, o que reflete o conjunto de condições que limita a extensão do nicho, o grau de tolerância e o sucesso de expansão das espécies no espaço (Abílio et al. 2006; Abílio et al. 2007; Lockwood et al. 2005; Simberlof 2009). 
Avaliação alométrica e da biomassa de Melanoides tuberculata (MULLER, 1774) como ferramenta para a avaliação da qualidade ambiental de reservatórios

\section{Conclusão}

A partir dos resultados obtidos, pode-se constatar que os tamanhos de M. tubeculata mostram relação com o grau de eutrofização dos locais de amostragem. Contudo, estudos que considerem a metodologia aplicada nesse trabalho, devem ser repetidos em reservatórios que apresentem maior amplitude no gradiente trófico, tendo em vista avaliar se os padrões encontrados nesse estudo se repetem em outros ambientes.

\section{Agradecimentos}

Os autores agradecem a FINEP (Agência Brasileira de Inovação) pelo financiamento do projeto de pesquisa MCTI/ FINEP CT-HIDRO 01/2013 (1107/13 - BIOQUALI: Produtos e processos para o biomonitoramento da qualidade da água, dinâmica dos corpos hídricos e hidrologia da bacia representativa do Rio Taperoá) que resultou na elaboração desse trabalho. A Coordenação de Aperfeiçoamento de Pessoal de Nível Superior (CAPES) pela bolsa de Pós-doutorado Júnior (processo 88887.374939/2019-00) a DJA; pela bolsa de pesquisa de produtividade (processo 302393/2017-0) concedida a JM. Ao Laboratório de Ecologia de Bentos e Laboratório de Ecologia Aquática pelo suporte no processamento das amostras.

Participação dos autores: MA - Idealização e conceitualização do trabalho, metodologia e análise formal; ELA, DJZ Metodologia, redação, revisão e edição; JELB - Redação, revisão e edição; JM - Coordenação e supervisão geral do artigo, metodologia, análise de dados, redação, revisão, e edição.

Aprovação ética ou licenças de pesquisa: trabalho dispensado de licenças.

Disponibilidade dos dados: os dados não estão depositados em nenhuma base.

Fomento: MCTI/ FINEP CT-HIDRO 01/2013 (1107/13 - BIOQUALI: Produtos e processos para o biomonitoramento da qualidade da água, dinâmica dos corpos hídricos e hidrologia da bacia representativa do Rio Taperoá.

Conflito de interesses: Os autores declaram não haver interesses financeiros ou pessoais que possam exercer influências sobre o conteúdo apresentado nesse trabalho.

\section{Referências}

Abílio FJP, Ruffo TLM, Souza AHFF, Florentino HS, Oliveira-Júnior ET, Meireles BN, Santana ACD. 2007. Macroinvertebrados Bentônicos como bioindicadores de qualidade ambiental de corpos aquáticos da Caatinga. Oecologia Brasiliensis, 11: 397-409.

Abílio FJP, Fonseca-Gessner AA, Leite RL, Ruffu TRM. 2006. Gastrópodes e outros invertebrados do sedimento e associados à macrófita Eichhornia crassipes de um açude hipertrófico do semi-árido paraibano. Revista de Biologia e Ciências da Terra, 1: 65-178.

Anderson MJA. 2001. New method for non-parametric multivariate analysis of variance. Austral Ecology, 1:32-46.

Anderson MJ, Gorley RN, Clarke KR. 2008.PERMANOVA + forPRIMER: Guide to Software and Statistical Methods. Primer-e, Plymouth, UK, 214p.

APHA. 2005. Standard Methods for the Examination of Water and Wastewater. 21st Edition, American Public Health Association/American Water Works Association/Water Environment Federation, Washington DC. 
Araújo SMS. 2011. A região semiárida do nordeste do Brasil: questões ambientais e possibilidades de uso sustentável de recursos. Revista Científica da FASETE, 5: 88-98.

\section{AYRES M, AYRES DL, Santos AAS, AYRES-JUNIOR M, AYRES D, SANTOS A, AYRES M. 2007. BioEstat 5.0: aplicações estatísticas nas áreas das ciências biológicas e médicas.}

Azevêdo DJS, Barbosa JEL, Gomes WIA, Porto DE, Marques JC, Molozzi J. 2015. Diversity measures in macroinvertebrate and zooplankton communities related to the trophic status of subtropical reservoirs: Contradictory or complementary responses? Ecological Indicators, 50: 135-149.

Azevêdo EL, Alves RRN, Dias TLP, Molozzi J. 2017a. How do people gain access to water resources in the Brazilian semiarid (Caatinga) in times of climate change? Environmental monitoring and assessment, 89: 375.https://doi. org/10.1007/s10661-017-6087-z.

Azevêdo EL, Barbosa JEL, Viana LGV, Anacleto MJP, Callisto M, Molozzi J. 2017b. Application of a statistical model for the assessment of environmental quality in neotropical semi-arid reservoirs. Environmental Monitoring and Assessment, 189: 1-13. DOI 10.1007/s10661-016-5723-3.https://doi.org/10.1007/s10661-016-5723-3.

Barbosa, JEL, Medeiros, ESF, Brasil, J, Cordeiro, RS, Crisim, MCB, Silva, GHG. 2012. Aquatic systems in semi-arid Brazil: limnology and management. Acta Limnologica Brasiliensia.vol. 24, no 1, pag.103-118.https://doi.org/10.1590/ S2179-975X2012005000030

Barbour, M.T., J. Gerritsen, B.D. Snyder, and J.B. Stribling. 1999. Rapid Bioassessment Protocols for Use in Stream sand Wadeable Rivers: Periphyton, Benthic Macroinvertebrates and Fish, Second Edition.

Braga GG, Becker V, Oliveira JNP, Junior JRM, Bezerra AFM, Torres LM, Galvão AMF, Mattos A. 2015. Influência da estiagem prolongada na qualidade da água de reservatórios tropicais em uma região semiárida. Acta Limnologica Brasiliensia, 15-23. http://dx.doi.org/10.1590/S2179-975X2214

Da Costa MRA, Attayde JL, Backer V.2016. Effects of water level reduction on the dynamics of phytoplankton functional groups in tropical semi-arid shallow lakes. Hydrobiologia, 778:75-89. https://doi.org/10.1007/s10750-015-2593-6

De Sousa-Souto L, Brito MFG, ROSA LC. 2011. Melanoides tuberculatus (Müller, 1774): a new threat to the conservation of native aquatic species in Sergipe, Brazil. Scientia Plena, 7: 1-6.

Dodds WK, Smith VH. 2006. Nitrogen, phosphorus, and eutrophication in streams. Inland Waters, 6:155-164. https:// doi.org/10.5268/IW-6.2.909.

Drobac D, Tokodi N, Simeunović J, Baltić V, Stanić D, Stanić Z. 2013. Human exposure to cyanotoxin sand their effectsonh ealth. Arh Hig Rada Toksikol, 64:305-316. DOI: 10.2478/10004-1254-64-2013-2320

Fonseca JR, Vieira PCS, Kujibida P, Costa IAS. 2015. Cyanobacterial occurrence and detection of microcystins and saxitoxins in reservoirs of the Brazilian semi-arid. Acta Limnologica Brasiliensia, 27: 78-92. https://doi.org/10.1590/ S2179-975X2814

J.Liebe, N. Van de Giesen, M. Andreini. 2005. Estimation of small reservoir storage capacities in a semi-arid environment A case study in the Upper East Region of Ghana. Elsevier, 448-454.

Junior CANR, Costa MRA, Menezes RF, Attayde JL, Becker V. 2018. A redução do volume intensifica o risco a eutrofização em reservatórios do semiárido tropical. Acta Limnologica Brasiliensia, vol. 30, e 106. https://doi.org/10.1590/s2179$975 \times 2117$

Goulart, M. ;Callisto, M. 2003. Bioindicadores de qualidade de água como ferramenta em estudos de impacto ambiental. Revista da FAPAM.ano $2, \mathrm{n}^{\circ} 1$.

Liebe J, Van de Giesen N, Andreini M. 2005. Estimation of small reservoir storage capacities in a semi-arid environment A case study in the Upper East Region of Ghana. Physics and Chemistry of the Earth 30: 448-454, Doi:10.1016/j. pce.2005.06.011. 
Avaliação alométrica e da biomassa de Melanoides tuberculata (MULLER, 1774) como ferramenta para a avaliação da qualidade ambiental de reservatórios

Lockwood JL, Cassey P, Blackburn T. 2005.The role of propagule pressure in explaining species invasions. Trends in ecology \& evolution, 20: 223-228.

Lorenzi AS, Araújo MKC, Chia MA, Oliveira MCB. 2018. Cyanotoxin contamination of semiarid drinking water supply reservoirs. Environmental Earth Sciences, 77:595.https://doi.org/10.1007/s12665-018-7774-y

Macedo CF, Sipaúba-Tavares LH. 2010. Eutrofização e qualidade da água na piscicultura: consequências e recomendações. Boletim do Instituto de Pesca, 36: 149-163.

Menezes RF, Attayde JL, Kosten S, Lacerot G, Souza LC, Costa LS, Sternberg LSL, Santos AC, Rodrigues MM, Jeppesen E. 2019. Differences in food webs and trophic states of Brazilian tropical humid and semi-arid shallow lakes: implications of climate change. Hydrobiologia, 829: 95-111. https://doi.org/10.1007/s10750-018-3626-8.

Molozzi J, Salas F, Callisto M, Marques JC. 2013. Thermodynamic oriented ecological indicators: Application of EcoExergy and Specific Eco-Exergy in capturing environmental changes between disturbed and non-disturbed tropical reservoirs. Ecological Indicators, 24: 543-551. http://dx.doi.org/10.1016/j.ecolind.2012.08.002.

Mugnai R, Nessimian JL, Baptista DF. 2010. Manual de identificação de macroinvertebrados aquáticos do Estado do Rio de Janeiro. Technical Books Editora. 174p.

Oliveira CDL, DE OLIVEIRA CYB. 2019. Growth parameters of the invasive gastropod Melanoides tuberculata (Müller, 1774) (Gastropoda, Thiaridae) in a semi-arid region, Northeastern Brazil. Acta Scientiarum. Biological Sciences, 41: 1-9. https://doi.org/10.4025/actascibiolsci.v41i1.45720.

Olivera SLE, Ribeiro H. 2014. Variabilidade climática e qualidade da água do Reservatório Guarapiranga. Estudos avançados, 28: 95-128. https://doi.org/10.1590/S0103-40142014000300007.

Paiva FF, Gomes W, Medeiros CRF, Alvaro ELF, Ribeiro IMS, Molozzi J. 2018. Environmental factors influencing the occurrence of alien mollusks in semiarid reservoirs.Limnetica, 37: 187-198. DOI: 10.23818/limn.37.16.

POMPÊO, M. 2017. Monitoramento e manejo de macrófitas aquáticas em reservatórios tropicais brasileiros . Instituto de Biociências. http://dx.doi.org/10.11606/9788585658670

Queiroz JF, Silva MSGM, Strixino ST. 2008. Organismos bentônicos: biomonitoramento de qualidade de águas. Embrapa meio ambiente.

Raw JL, Perissinotto R, Miranda NAF, Peer N. 2016. Feeding dynamics of Melanoides tuberculata (Müller,1774). Journal of Molluscan Studies, 82: 328-335. https://doi.org/10.1093/mollus/eyv070.

Ren Y, Yang M, Chen M, Zhu Q, Zhou L, Qin W, Wang T. 2017. Microcystin-LR promotes epithelial-mesenchymal transition in colorectal câncer cell sthrough PI3-K/AKT and SMAD2. / Toxicology Letters, 53-60. https://doi. org/10.1016/j.toxlet.2016.11.004.

Reynaud A, Lanzanova D. 2017. A Global Meta-Analysis of the Value of Ecosystem Services Provided by Lakes. Ecological Economics 137: 184-194. https://doi.org/10.1016/j.ecolecon.2017.03.001.

Rezende RS, Novaes JLC, Albuquerque CQ, Costa RS, Junior JFG. 2018. Aquatic invertebrates increase litter break down in Neotropical shallow semi-arid lakes. Journal of Arid Environments, 8-14. https://doi.org/10.1016/j. jaridenv.2018.03.002

Salameh E, Harahsheh S. 2011. In: Ansari AA, Singh GS, Lanza GR, Rast W. Eutrophication: Causes, Consequences and Control. Springer Science \& Business Media, p.69-90.

Santos CM, Eskinazi-Sant'anna EM. 2010. The introduced snail Melanoidestuberculatus(Muller, 1774) (Mollusca: Thiaridae) in aquatic ecosystems of the Brazilian semiarid northeast (Piranhas-Assu River basin, State of Rio Grande do Norte). Brazilian Journal of Biology, 70: 1-7. https://doi.org/10.1590/S1519-69842010000100003. 
Simberloff D. 2009.The role of propagule pressure in biological invasions. Annual Review of Ecology, Evolution, and Systematics, 40: 81-102.

Trindade PBCB, Mendonça ASF. 2014. Eutrofização em Reservatórios: Estudo de Caso: Reservatório de Rio Bonito (ES). Engenharia Sanitária e Ambiental, 19: 275-282. https://doi.org/10.1590/S1413-41522014019000000537.

Van Apeldoorn ME, Van Egmond HP, Speijers GJA, Bakke GJI. 2007. Toxins of cyanobacteria. Mol. Nutr. Food Res, 51, 7 - 60. DOI 10.1002/mnfr.200600185

Vasconcelos JF, Barbosa JEL, Azevedo EL, Azevedo DJS, Anacleto MJP. 2013. Predation effects of Melanoides tuberculata (Müller 1774) on periphytic biofilm colonization: an experimental approach. Biota Neotropica, 13: 96-101. https://doi. org/10.1590/S1676-06032013000200010.

Wiegand MC, Piedra JIG, Araújo JC. 2016. Vulnerabilidade à eutrofização de dois lagos tropicais de climas úmido (Cuba) e semiárido (Brasil). Engenharia Sanitária e Ambiental, 21: 415-424. https://doi.org/10.1590/s1413-41522016139527.

Zhou L, Yu H, Chen K. 2002. Relationship between microcystin in drinking water and colorectal cancer. Biomed Environ Sci, 15(2):166-71. PMID: 12244757. 